\title{
DISTRIBUCIÓN DIFERENCIAL DE DOS ESPECIES DE MUÉRDAGO ENANO SOBRE PINUS HARTWEGII EN EL ÁREA NATURAL PROTEGIDA “ZOQUIAPAN Y ANEXAS”, ESTADO DE MÉXICO
}

\author{
Mónica E. Queijeiro-Bolaños ${ }^{1}$, Zenón Cano-Santana \\ e IVÁN CASTELlanos-Vargas \\ Universidad Nacional Autónoma de México, Facultad de Ciencias, Departamento \\ de Ecología y Recursos Naturales, 04510 México, D.F. México. \\ 1Autor para la correspondencia: monicq15@yahoo.com
}

\begin{abstract}
RESUMEN
Los muérdagos enanos (Arceuthobium spp.) son plantas importantes en los bosques templados de México debido a su alta incidencia. Al sur del cerro Papayo en el Área Natural Protegida "Zoquiapan y Anexas", Estado de México, coexisten Arceuthobium vaginatum subsp. vaginatum y $A$. globosum subsp. grandicaule incluso en el mismo árbol hospedero de la especie Pinus hartwegii. Al estudiar su disposición vertical en el tronco se registra que A. vaginatum se distribuye a menor altura en los pinos que A. globosum. Esto sugiere que la eficiencia de obtención de recursos nutricionales del hospedero podría ser diferencial, por lo que es posible que haya una relación de competencia entre ambas especies.
\end{abstract}

Palabras clave: Arceuthobium, distribución vertical, muérdago enano, Pinus hartwegii.

\section{ABSTRACT}

Dwarf mistletoes (Arceuthobium spp.) are important plants in temperate forests of Mexico due to their high incidence. At the south side of the Papayo Hill in the "Zoquiapan y Anexas" Natural Protected Area, Mexico State, both, Arceuthobium vaginatum subsp. vaginatum and A. globosum subsp. grandicaule coexist within the same zone and, frequently, on the same host tree of the species Pinus hartwegii. We studied the vertical distribution of both species on the stem, and we registered that $A$. vaginatum is found more frequently to lower heights of the pines than A. globosum. These results suggest that the nutritional 
resources are taken in a differential manner, causing a probable competitive relation between the two species.

Key words: Arceuthobium, dwarf mistletoe, Pinus hartwegii, vertical distribution.

\section{INTRODUCCIÓN}

Las plantas parásitas son un grupo polifilético de amplia distribución mundial (Musselman y Press, 1995; Nickrent, 2002). En México se encuentran con gran frecuencia los del género Arceuthobium (Viscaceae), conocidos comúnmente como muérdagos enanos; se trata de plantas arbustivas hemiparásitas de coníferas, de menos de $50 \mathrm{~cm}$ de altura y con un color amarillento, rojizo o negruzco, que presentan hojas inconspicuas y escuamiformes y flores unisexuales (Rzedowski y Rzedowski, 2001; Agrios, 2005; Mathiasen et al., 2008).

Los muérdagos enanos constituyen la segunda causa de daño a los bosques de coníferas de México después de los incendios (Hawksworth, 1983), y el segundo lugar de daño biológico, después de los insectos descortezadores (Madrigal et al., 2007), ocasionando una pérdida anual de hasta $1.4 \mathrm{~m}^{3} / \mathrm{año}^{-1} \mathrm{ha}^{-1}$ de superficie de bosque (Vázquez et al., 2006), ya que afectan la supervivencia y crecimiento de las coníferas infectadas (Gutiérrez, 1968; Andrade y Cibrián, 1980; Madrigal et al., 2007).

La mayor parte de los representantes del género se distribuyen en América donde están presentes 18 de los 26 existentes (según la filogenia a partir de ADN nuclear y de cloroplasto) y su área global se extiende desde el oeste de Estados Unidos hasta Centro América (Hawksworth et al., 2002; Nickrent et al., 2004). En México se encuentran 14 especies de muérdago enano distribuidas en 24 estados (Hawksworth y Wiens, 1996; Nickrent et al., 2004). Existen reportes recientes que documentan incrementos en los niveles de incidencia (porcentaje de árboles infestados) y de su área de distribución tanto de $A$. globosum como de $A$. vaginatum en diversas zonas de México y de algunos países de América Central (Melgar et al., 2001; Mathiasen et al., 2004; Mathiasen, 2007).

El ciclo de vida del muérdago enano se divide básicamente en cuatro fases, que son: dispersión, que es la expulsión de tipo balístico de las semillas; establecimiento, que comprende desde la llegada de la semilla al hospedero hasta la germinación; incubación, que es cuando se desarrolla el sistema endofítico; y reproducción, que se inicia con la formación de tallos aéreos con flores (Hawksworth et al., 2002; Mathiasen et al., 2008). 
La supervivencia y la fecundidad de los muérdagos enanos son afectadas por el clima, sobre todo por la temperatura y la humedad (Hawksworth y Wiens, 1996), en tanto que su incidencia lo es por la altitud y la pendiente del terreno (QueijeiroBolaños, 2007), ya que se encuentran más comúnmente en lugares con pendientes moderadas (de menos de 10\%) que en aquellas pronunciadas (Hawksworth y Wiens, 1996), sin embargo esto varía según el sitio.

Se han hecho numerosas observaciones acerca de la presencia de muérdagos enanos sobre Pinus hartwegii Lindl. (Pinaceae) en México, muchas de las cuales han sido llevadas a cabo en el Área Natural Protegida "Zoquiapan y Anexas" (ANPZ), Estado de México. Se reporta, por ejemplo, la reducción del crecimiento y la baja fecundidad en pinos atacados por estas plantas (Gutiérrez, 1968; Andrade y Cibrián, 1980; Madrigal et al., 2007). Además, se registra una correlación positiva entre la probabilidad y nivel de infección y la talla de los árboles (Arriaga et al., 1988; Hernández-Benítez et al., 2005). En octubre de 2004 se encontró que en una zona cercana al cerro Papayo en el ANPZ A. globosum subsp. grandicaule Hawks. \& Wiens parasita hasta $70 \%$ de los árboles de $P$. hartwegii del lugar (HernándezBenítez et al., 2005), en tanto que, en julio de 2005 Queijeiro-Bolaños (2007) reporta la presencia de A. vaginatum subsp. vaginatum (Willd.) Presl en la misma zona. En este último estudio se relacionó la incidencia de ambos muérdagos con seis indicadores de disturbio propios del ANPZ (áreas quemadas, presencia de boñigas, basura, zonas denudadas, densidad de árboles muertos y árboles talados), pero no se presentaron correlaciones significativas de ninguno de éstos sobre la incidencia de ambos muérdagos; sin embargo, se encontró una negativa entre los niveles de incidencia de las dos especies de muérdago dentro del ANPZ, por lo que se ha sugerido la existencia de competencia entre ellas.

Los estudios que examinan las interacciones simultáneas entre las plantas hospederas y dos o más especies de parásitos son raros (ver, p. ej., Linhart et al., 1994) y no existen reportes de este tipo para muérdagos enanos. Sin embargo, el tema resulta relevante, ya que tales parásitos cumplen un papel importante en la dinámica de la comunidad que habitan, ya que los árboles infectados por Arceuthobium son debilitados y facilitan la infección de otros parásitos (Linhart et al., 1994; Watson, 2001).

En la búsqueda de entender la ecología de estos dos muérdagos enanos, el objetivo del presente estudio es conocer cómo es la distribución vertical de $A$. vaginatum subsp. vaginatum y A. globosum subsp. grandicaule sobre los árboles de $P$. hartwegii en una zona del ANPZ para determinar si existe un patrón diferencial. 


\section{MATERIALES Y MÉTODOS}

Área de estudio

El Área Natural Protegida "Zoquiapan y Anexas” (ANPZ) se localiza en el municipio de Tlalmanalco, Estado de México. Tiene una extensión de 19,400 ha y forma parte de la Región Terrestre Prioritaria "Sierra Nevada" (Obieta y Sarukhán, 1981; Rojas, 1983; Arriaga et al., 2000). La estación meteorológica aledaña de Río Frío registra una temperatura media anual de $9.7^{\circ} \mathrm{C}$ y una precipitación anual de $941 \mathrm{~mm}$, con las lluvias concentradas entre junio y septiembre. El clima del sitio es templado subhúmedo con lluvias en verano y una elevación que varía de 2850 a 4150 $\mathrm{m}$ (Vargas, 1997). El estudio se realizó en una parcela de $150 \times 200 \mathrm{~m}$ de bosque abierto de $P$. hartwegii localizada al sur del cerro Papayo, en la parte sureste del ANPZ (19² 18'08.4" N, 98 $42^{\prime} 10.7^{\prime \prime} \mathrm{O}$ a 3420 m s.n.m.), dentro de un terreno plano sin pendiente, en el cual se hallan algunos elementos de Alnus jorullensis Kunth subsp. jorullensis (Betulaceae). En el sotobosque se desarrollan gramíneas de los géneros Muhlenbergia, Festuca, Stipa y Piptochaetium y otras hierbas y arbustos como Lupinus campestris Cham. et Schltdl., Achillea millefollium L., Penstemon campanulatus (Cav.) Willd. y Senecio cinerarioides Kunth, entre otras (ver detalles en Hernández-Benítez et al., 2005).

Muestreo

En marzo de 2006 se seleccionaron por vagabundeo aproximadamente 110 individuos de $P$. hartwegii que presentaban infestación por una o las dos especies de muérdago enano. En cada uno de ellos se midió la altura a la que se distribuían todas las plantas de Arceuthobium que se asentaban a lo largo del tronco y sobre las ramas. La medición de su altura sobre el pino se hizo con un flexómetro a partir del suelo y hasta el punto de inserción de la planta. Para aquellos muérdagos que crecían por arriba de los $3 \mathrm{~m}$, la altura del punto de inserción fue estimada obteniendo el ángulo $\alpha$ con ayuda de un clisímetro y la distancia del observador al objeto $(d)$. Tal medida (h) se obtuvo con la ecuación $h=d \times \tan \alpha$.

Análisis estadístico

Los datos obtenidos se analizaron mediante una prueba de $\chi^{2}$ de independencia, con la cual se comparó la distribución de frecuencias de altura de ambas 
especies de muérdago. También se hizo una prueba de $t$ para comparar la altura promedio a la que se distribuyen las dos plantas parásitas sobre el pino. Los resultados se obtuvieron con el programa STATISTICA versión 6.0 (Statsoft, 2001).

\section{RESULTADOS}

La altura promedio a la que se distribuyen ambas especies de muérdago sobre el pino fue significativamente diferente $(t=12.23$, g.l. $=1338, P<0.0001) ;$ A. globosum se encuentra con mayor frecuencia en zonas más elevadas del tronco del hospedero $(2.93 \pm$ e.e. $0.11 \mathrm{~m}$, mediana $=2.00 \mathrm{~m}, n=727)$ que $A$. vaginatum $(1.34 \pm 0.05 \mathrm{~m}$, mediana $=1.06 \mathrm{~m}, n=613)$. La primera se presenta con mayor frecuencia entre $0.01 \mathrm{y}$ $1.71 \mathrm{~m}$, mientras que la segunda lo hace entre 0.10 y $5.13 \mathrm{~m}\left(\chi^{2}=160.26\right.$, g.l. $=13, P<$ 0.0001; Fig. 1).



Fig. 1. Distribución de alturas a las que se encuentran los muérdagos $A$. vaginatum y $A$. globosum sobre P. hartwegii en el Área Natural Protegida "Zoquiapan y Anexas". Datos de marzo de 2006. Las distribuciones de frecuencias fueron significativamente diferentes $\left(\chi^{2}=\right.$ 160.26, g.l. $=13, P<0.0001)$. El intervalo de distribución en el que se encuentra $A$. globosum es de 0.1 a $23.9 \mathrm{~m}$ y $A$. vaginatum se encuentra de 0.01 a $9.9 \mathrm{~m}$.

\section{DISCUSIÓN Y CONCLUSIONES}

Las dos especies de muérdago enano presentan una distribución diferencial sobre el tallo de los pinos de $P$. hartwegii. Arceuthobium vaginatum se caracteriza 
por tener sus tejidos de color café oscuro a negro, se distribuye con más frecuencia en las partes bajas y sombreadas de los árboles hospederos, en tanto que $A$. globosum, que tiene tejidos amarillos a amarillo-verdosos, crece en las porciones más altas y soleadas. Esta observación sugiere que A. vaginatum podría tener una menor capacidad fotosintética (y menor requerimiento de radiación solar) que $A$. globosum; hecho que debe ser corroborado mediante el uso de aparatos. Es notable que la distribución de $A$. vaginatum en las partes inferiores de los árboles sea contrastante con la observación general de que la infección por plantas parásitas es mucho más frecuente en árboles de estatura elevada, ya que en alturas bajas hay una menor asimilación de carbono por el efecto de sombreado del hospedero (Graves, 1995; Shaw y Weiss, 2000; Bickford y Kolb, 2005); sin embargo, A. vaginatum puede obtener una ventaja sobre $A$. globosum al tener acceso de manera más directa a las reservas del hospedero cercanas a la raíz, sobre todo considerando que tendría menor capacidad para producir fotosintatos, con lo cual compensaría la desventaja del sombreado.

Por otro lado, este resultado complementa las observaciones de un estudio paralelo (Queijeiro-Bolaños, 2007) en el que, mediante un análisis de regresión múltiple realizado con datos de 24 parcelas del ANPZ para verificar el efecto de diversas variables de disturbio y ambientales sobre la frecuencia de árboles infestados por ambos muérdagos, se encuentra que (1) la incidencia de $A$. vaginatum fue afectada positivamente por la pendiente del terreno y negativamente por la frecuencia relativa de $A$. globosum, y (2) la incidencia de A. globosum fue influida de manera positiva por la altitud y la densidad de árboles no hospederos, y negativamente por la frecuencia relativa de $A$. vaginatum. Una causa de esta distribución diferencial podría ser el hecho de que ambas especies están compitiendo entre sí por los recursos nutritivos que obtienen de su hospedero.

Arceuthobium globosum es hasta ahora la única especie de muérdago enano en la que se ha reportado floema en los tallos aéreos, mientras que éste se encuentra ausente en los miembros del género con mayor especificidad al hospedero (Calvin et al., 1984; Stewart y Press, 1990; Nickrent y Musselman, 2004). Como parte del hábito parásito, los muérdagos muestran modificaciones anatómicas, como la ausencia de floema, por lo que acumulan grandes cantidades de fotosintatos en el sistema endofítico y éstos se conducen hacia la parte aérea por otro medio diferente (Hull y Leonard, 1964a, b; Calvin et al., 1984). Se considera que los muérdagos enanos son plantas hemiparásitas con una capacidad fotosintética reducida, y se ha encontrado que pueden obtener hasta $30 \%$ de los fotosintatos del hospedero (Hull y Leonard, 1964b; Hibberd y Jeschke, 2001). 
A pesar de los resultados obtenidos, así como de los datos de Queijeiro-Bolaños (2007), los cuales sugieren la existencia de una interacción competitiva entre ambas especies de muérdagos, es necesario llevar a cabo trabajos experimentales que lo prueben. Asimismo, es deseable realizar estudios sobre la fisiología de las dos especies y de sus respuestas a los factores abióticos (Shaw y Weiss, 2000) y bióticos. Esta contribución muestra que dos especies de Arceuthobium que explotan los mismos recursos pueden tener como estrategia competitiva la distribución vertical diferencial sobre el hospedero y plantea nuevas perspectivas de investigación, principalmente sobre el aspecto ecofisiológico de los mismos.

\section{LITERATURA CITADA}

Agrios, G. 2005. Plant pathology. Elsevier Academic Press. Londres. 922 pp.

Andrade, V. y D. Cibrián T. 1980. Evaluación de poblaciones de muérdago enano (Arceuthobium globosum Hawks. y Wiens y A. vaginatum Willd.) en bosques de Pinus hartwegii Lindl. en Zoquiapan, Edo. de México. Sociedad Mexicana de Entomología. In: Memoria primera. Simposio Nacional sobre Parasitología Forestal. 18 y 19 de febrero de 1980. Uruapan, Michoacán. pp. 238-253.

Arriaga, L., M. Franco y J. Sarukhán. 1988. Identification of natural groups of trees of uneven-aged forest using multivariate methods. J. Ecol. 76: 1092-1100.

Arriaga, L., J. M. Espinoza, C. Aguilar, E. Martínez, L. Gómez y E. Loa (coords.). 2000. Regiones terrestres prioritarias de México. Comisión Nacional para el Conocimiento y Uso de la Biodiversidad. México, D.F. 609 pp.

Bickford, C. y T. Kolb. 2005. Host physiological condition regulates parasitic plant performance: Arceuthobium vaginatum subsp. cryptopodum on Pinus ponderosa. Oecologia 146: 179-189.

Calvin, C., F. G. Hawksworth y D. M. Knutson. 1984. Phloem in Arceuthobium globosum (Viscaceae). Bot. Gaz. 145: 461-464.

Graves, J. 1995. Host-plant responses to parasitism. In: Press, M. y J. Graves (eds.). Parasitic plants. Chapman \& Hall. Londres. pp. 206-225.

Gutiérrez, R. M. 1968. Efecto del parasitismo del muérdago enano (Arceuthobium spp.) sobre el desarrollo en grosor del tronco de Pinus montezumae y P. hartwegii en el cerro Telapón, Estado de México. Tesis profesional. Facultad de Ciencias, Universidad Nacional Autónoma de México. México, D.F. 28 pp.

Hawksworth, F. G. 1983. Mistletoes as forest parasites. In: Calder, M. y P. Bernhardt (eds.). The biology of mistletoes. Academic Press. Nueva York. pp. 320-329.

Hawksworth, F. G. y D. Wiens. 1996. Dwarf mistletoes: Biology, pathology and systematics. Agriculture Handbook 709. United States Department of Agriculture, Forest Service. Washington, D.C. 410 pp. 
Hawksworth, F. G., D. Wiens y B. W. Geils. 2002. Arceuthobium in North America. In: Geils, B. W., J. Cibrián T. y B. Moody (eds.). Mistletoes of North American conifers. Reporte Técnico General RMRS-GTR-98. United States Department of Agriculture, Forest Service. Fort Collins. pp. 29-56.

Hernández-Benítez, R., Z. Cano-Santana e I. Castellanos-Vargas. 2005. Incidencia de infestación de Arceuthobium globosum grandicaule (Hawks. y Wiens) en Pinus hartwegii (Lindl.). Cienc. For. Méx. 30: 79-86.

Hibberd, J. y D. Jeschke. 2001. Solute flux into parasitic plants. J. Exp. Bot. 52: 2043-2049.

Hull, R. J. y O. A. Leonard. 1964a. Physiological aspects of parasitism in mistletoes (Arceuthobium and Phoradendron). I. The carbohydrate nutrition of mistletoe. Plant Physiol. 39: 996-1007.

Hull, R. J. y O. A. Leonard. 1964b. Physiological aspects of parasitism in mistletoes (Arceuthobium and Phoradendron). II. Photosynthetic capacity of mistletoe. Plant Physiol. 39: 1008-1017.

Linhart, Y. B., M. A. Snyder y J. P. Gibson. 1994. Differential host utilization by two parasites in a population of ponderosa pine. Oecologia 98: 117-120.

Madrigal, S., I. Vázquez y E. Velasco. 2007. Obtención de parámetros dasométricos para evaluar efecto causado por Arceuthobium vaginatum en Pinus hartwegii del Nevado de Colima. Memorias del VII Congreso Mexicano de Recursos Forestales. 28 al 31 de octubre de 2005. Morelia, Michoacán. pp. 1-7.

Mathiasen, R., A. Flores, H. Miranda y L. Cadio. 2004. First report of Arceuthobium vaginatum subsp. vaginatum on Pinus pseudostrobus. Plant Dis. 88: 1046.

Mathiasen, R. 2007. First report of Durangan dwarf mistletoe, Arceuthobium vaginatum subsp. durangense, on Pinus cooperi and P. engelmannii in Mexico. Plant Dis. 91: 1201.

Mathiasen, R. L., D. L. Nickrent, D. C. Shaw y D. M. Watson. 2008. Mistletoes. Pathology, systematics, ecology and management. Plant Dis. 92: 988-1006.

Melgar, J., R. Mathiasen y B. Howell. 2001. First report of Arceuthobium globosum subsp. grandicaule in Honduras. Plant Dis. 85: 563.

Musselman, L. J. y M. C. Press. 1995. Introduction to parasitic plants. In: Press, M. C. y J. D. Graves (eds.). Parasitic plants. Chapman \& Hall. Londres. pp. 1-13.

Nickrent, D. L. 2002. Orígenes filogenéticos de las plantas parásitas. In: López-Sáez, J. A., P. Catalán y L. Sáez (eds.). Plantas de la península Ibérica e islas Baleares. MundiPrensa. Madrid. pp. 29-56.

Nickrent, D. L., M. A. García, M. P. Martín y R. L. Mathiasen. 2004. A phylogeny of all species of Arceuthobium (Viscaceae) using nuclear and chloroplast DNA sequences. Am. J. Bot. 91: 125-138.

Nickrent, D. L. y L. J. Musselman. 2004. Introduction to parasitic flowering plants. APS Education Center Introductory Topics: Introductions to the major pathogen groups. Consultado el 11 de marzo de 2010. http://www.apsnet.org/education/IntroPlantPath/ PathogenGroups/Parasiticplants/

Obieta, M. C. y J. Sarukhán. 1981. Estructura y composición de la vegetación herbácea de un bosque uniespecífico de Pinus hartwegii. I. Estructura y composición florística. Bol. Soc. Bot. Méx. 41: 75-124. 
Queijeiro-Bolaños, M. E. 2007. Interacciones entre dos especies de muérdago enano (Arceuthobium spp.) y Pinus hartwegii en el Parque Nacional Zoquiapan, Estado de México: el papel del disturbio. Tesis profesional. Facultad de Ciencias, Universidad Nacional Autónoma de México. México, D.F. 50 pp.

Rojas, M. C. 1983. Distribución de Arbutus spp. y su relación con Abies religiosa, Alnus firmifolia y Pinus spp. en la Estación Experimental Zoquiapan. Tesis profesional. Facultad de Ciencias, Universidad Nacional Autónoma de México. México, D.F. 101 pp.

Rzedowski, G. C. y J. Rzedowski. 2001. Flora fanerogámica del Valle de México. Instituto de Ecología, A.C. Pátzcuaro, Michoacán. 1406 pp.

Shaw, D. y S. Weiss. 2000. Canopy light and the distribution of hemlock dwarf mistletoe (Arceuthobium tsugense (Rosendahl) G.N. Jones subsp. tsugense) aerial shoots in an old-growth Douglas-fir/western hemlock forest. Northwest Science 74: 306-315.

Statsoft. 2001. Statistica 6.0. Statsoft Inc. Oklahoma.

Stewart, G. R. y M. C. Press. 1990. The physiology and biochemistry of parasitic angiosperms. Annu. Rev. Plant Physiol. Plant Mol. Biol. 41: 127-151.

Vargas, M. F. 1997. Parques Nacionales de México. Vol. I. Zonas Centro, Occidente y Oriente. Instituto Nacional de Ecología. México, D.F. 331 pp.

Vázquez C., I., A. Villa R. y S. Madrigal H. 2006. Los muérdagos (Loranthaceae) en Michoacán. Libro Técnico No. 2. Centro de Investigación Regional del Pacífico Centro, Instituto Nacional de Investigaciones Forestales, Agrícolas y Pecuarias. Uruapan, Michoacán. 98 pp.

Watson, D. M. 2001. Mistletoe - A keystone resource in forest and woodland worldwide. Annu. Rev. Ecol. Syst. 32: 219-249. 